\title{
DYNAMICS AND STABILITY: A LONG HISTORY FROM EQUILIBRIUM TO DYNAMICAL INTEGRITY
}

\author{
STEFANO LENCI, (*) DILETTA MARACCI (*)
}

SuNTO. - Il lavoro si pone l'obiettivo di raccontare una personale, e pertanto parziale, storia della dinamica e della stabilità nell'ambito della meccanica classica. L'idea è quella di introdurre i concetti attraverso degli esempi chiari e comprensibili, senza utilizzare formule matematiche. A tal fine si è scelto di prendere in considerazione alcuni fenomeni dinamici con cui siamo abituati a convivere e che potrebbero sembrare banali ma che, invece, sono in grado di spiegare concetti molto più complessi. Si parte da un breve riassunto dell'evoluzione del concetto di equilibrio, per poi passare alla discussione sulla stabilità e arrivare ai concetti più avanzati di stabilità strutturale e integrità dinamica.

$* * *$

ABSTRACT. - This work is aimed at reporting a personal, and thus partial, history of the dynamics and stability concepts within the framework of classical mechanics. The idea is to introduce these concepts without using mathematics and formula, but rather through practical and clear examples. For this purpose, it has been chosen to take in consideration some simple dynamic phenomena that we are used to live with, but that are able to explain more complex concepts. The paper starts with a brief summary on equilibrium concept evolution, passes through stability problems and arrives to more advanced concepts, such as structural stability and dynamical integrity.

\section{INTRODUCTION AND FRAMEWORK}

Dynamics is everywhere in our day life, which is plenty of movements and actions. Dynamics is life: we can imagine motion without life, but it is extremely difficult to figure out life without mobility. It is perhaps because of these reasons, or others, that incontrovertibly dynamics attracted the curiosity of human beings since long time ago,

(*) Università Politecnica delle Marche, Ancona, Italia. E-mail: lenci@univpm.it 
well before the scientific era, when it became one of the most important sciences. While losing its aura of "main science" it had in the past, in favor of new disciplines from which the humanity has many expectations (biology, information technology, economics, etc.), dynamics is still alive, still permeates our life, and it is still able to contribute to improve the quality of our lives. And it is not yet fully understood, nor fully exploited, although a lot of progresses have been done.

Dynamics has a long history of discoveries, successes and fails, attempts and chances, theory and experiments. It is not possible to quote all of them, but it is possible to select some and try to narrate a logical evolution of ideas and tools. This requires a choice, which is certainly personal, subjective and, thus, partial and incomplete. But it can help in delineating the main steps from the basic (and pioneering, according to the age in which they have been discovered) concepts to the modern developments up to the challenges for the future.

This is the goal of this work, which is based on Newtonian (or classical) mechanics (Isaac Newton, 1642-1727), which roughly descends from the well-known equation force $=$ mass $\cdot$ acceleration obtained from the second law of motion [1], although much more has been discovered and added to this basic formula. It is worth noting that in the $18^{\text {th }}$ and $19^{\text {th }}$ centuries, more abstract and general methods were developed, extending Newton's work and leading to two important reformulations known as Lagrangian and Hamiltonian mechanics [2, 3]. However, while being fundamental from many points of view, they do not add novelties to the basic assumptions of Newtonian mechanics, and provide "only" a different, rigorous and useful formalism.

Focusing on classical mechanics does not mean that the Relativity Theory and Quantum Mechanics are less important, nor that they do not have the phenomena we are going to discuss: simply, classical mechanics is sufficient for our illustrative purposes, especially because it is the one we experience in our everyday life.

Moreover, the dynamic phenomena discussed in this paper are not peculiar of mechanics, and are indeed important in many fields of engineering, science, economics, medicine, etc.

The work starts with an overview of the equilibrium concept evolution, and then deals with stability problems, giving a brief explanation of the bifurcation theory by means of some examples. Finally, the problems of structural stability and dynamical integrity are introduced and discussed. 


\section{EQUILIBRIUM}

The first dynamical issue that has been investigated in the past was equilibrium, i.e. lack of motion. The concept of equilibrium has its roots in the Greek scientific-philosophical thought. Science and philosophy, in fact, have been always strictly connected until the modern and sectorial division of disciplines. Particularly, Mechanics became a formal and axiomatic science only in the XVII century, and until then it corresponded essentially to Statics. The latter is one of the most ancient sciences, taking its origin from Greeks, who anticipated the concepts of gravity, force, equilibrium, motion, etc. [4, 5]. In this context, the first evidence we have about a scientific formulation of the equilibrium concept comes from the work of Archimedes (around 287-212 BC) [6]. In fact, he gave a rigorous explanation to the principle involved into the levers functioning, previously introduced by the Peripatetic School of Aristoteles' followers. In this context, Archimedes remarked, according to Pappus of Alexandria, "Give me

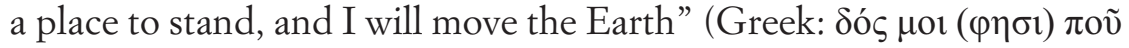

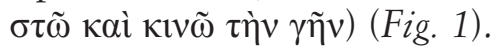

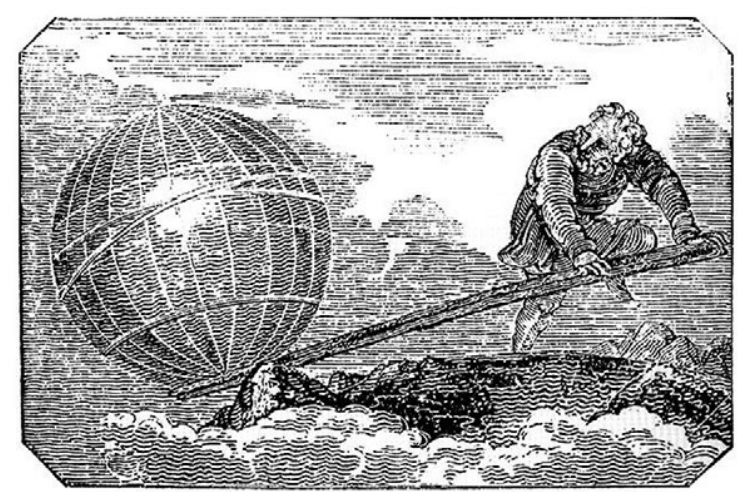

Fig. 1. The famous remark of Archimedes, quoted by Pappus of Alexandria:

"Give me where to stand, and I will move the Earth".

1 Quoted by Pappus of Alexandria, Collection or Synagoge, Book VIII, c. AD 340. Greek text: Pappi Alexandrini Collectionis, Edited by Friedrich Otto Hultsch, Berlin, 1878. 
Archimedes' work provided the basis of the equilibrium concept that, accordingly to subsequent mechanical theories, will be formulated as: 'a body is said to be in equilibrium when the sum of all forces (and angular momentum) acting on it is equal to zero'.

Other evidences that the equilibrium concept had already been well understood by Greeks derive from observing classical art and architecture. For instance, the low relief of the Parthenon (Fig. 2), conceived and drawn by Fidia, can be examined with graphical statics, building the funicular polygon of gravity forces and observing that the resultant falls into the base support [7].
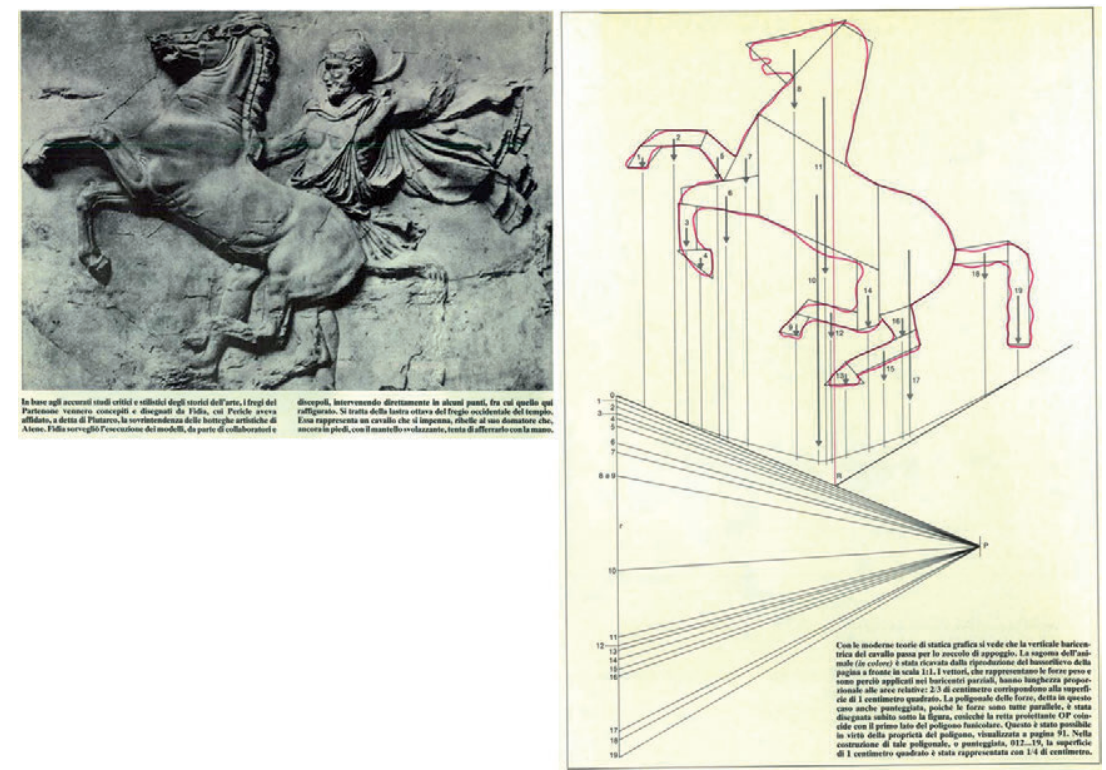

Fig. 2. The static conception of the Parthenon low relief conceived by Fidia [7].

The cultural heritage of theoretical mechanics received from the antiquity, as mentioned above, is focused on two big themes. The first one is related to the Aristotelian dynamics and, in particular, to its main axiom that establishes the proportionality between the "power" of a motion and its acquired velocity [8]. The second theme, instead, is the one that began with Archimedes' work on the gravity concept and lever functioning [9]. Later, in the Middle Ages and in the Renaissance many others (e.g. Giordano Nemorario (sec. XIII), 
Leonardo da Vinci (1452-1519)) contributed significantly to the development of the concepts of gravity, equilibrium, kinematics and dynamics. Obviously, one of the most important scientist working also on these arguments was Galileo (1564-1642), which can be considered the precursor of the Newtonian mechanics. In his essay De Motu, Galileo introduced his first ideas in the field of hydrostatics, statics and dynamics, through the study of solid bodies immersed in a fluid. All these ideas were then developed in the essay Discorso al Serenissimo Don Cosimo II, Gran Duca di Toscana, intorno alle cose che stanno in su l'acqua, o che in quella si muovono, published in Florence in 1612, which contains likely for the first time the definition of 'angular momentum' (Fig. 3), one of the most relevant concepts in Statics [10, 11]. Other important contributions of Galileo to Mechanics are focused on the inclined plane, on gravity and on pendulum. In particular, he claimed that a simple pendulum is isochronous, i.e. that its swings always take the same amount of time, independently of the amplitude [12], which is true if we are dealing with sufficiently small oscillations.

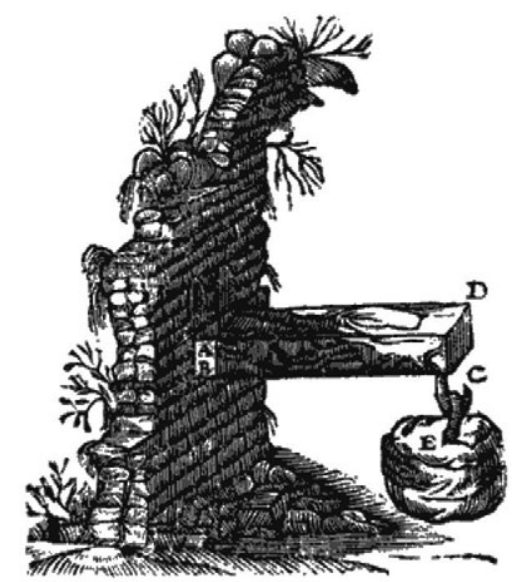

Fig. 3. Galileo's problem of cantilever beam [11].

A significant step forward in the 'problem of equilibrium' was made by Pierre Varignon (1654-1722) with the definition of the forces resultant (Fig. 4). He introduced also the 'parallelogram rule' and the 'Varignon's theorem', which permits to analytically calculate the center of mass position [13]. 


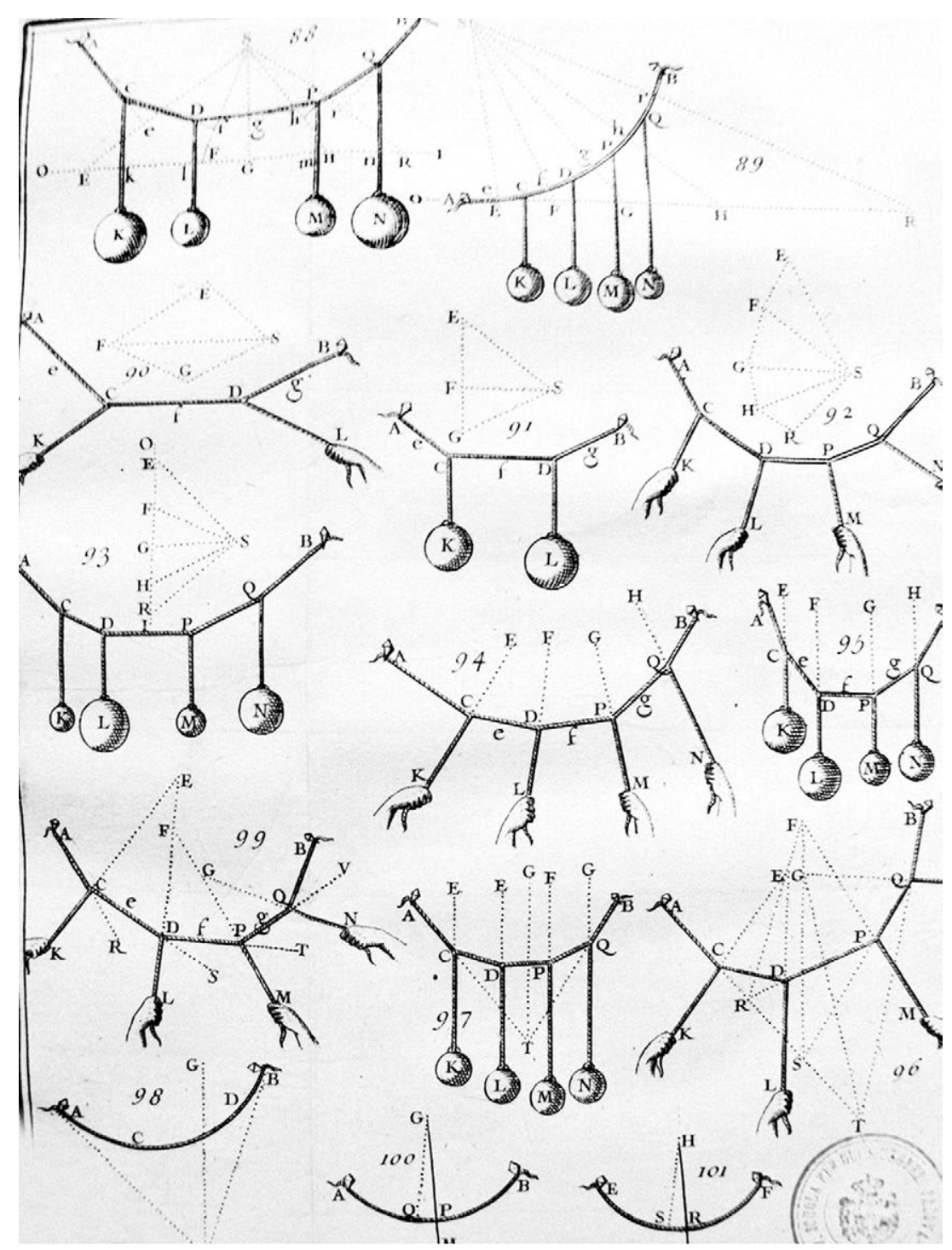

Fig. 4. Varignon's funicular polygon table [13].

Varignon's work contributed to lay the basis for what would be then called graphic (or graphical) statics. In graphical statics, the forces acting on a system are drawn into a force diagram, which is directly 
linked to their corresponding force polygons through geometrical constraints.

The use of these graphical methods to solve engineering problems is generally attributed to the German engineer Karl Culmann (1821-1881), who is considered the father of graphical statics (Fig. 5) $[14,15]$.
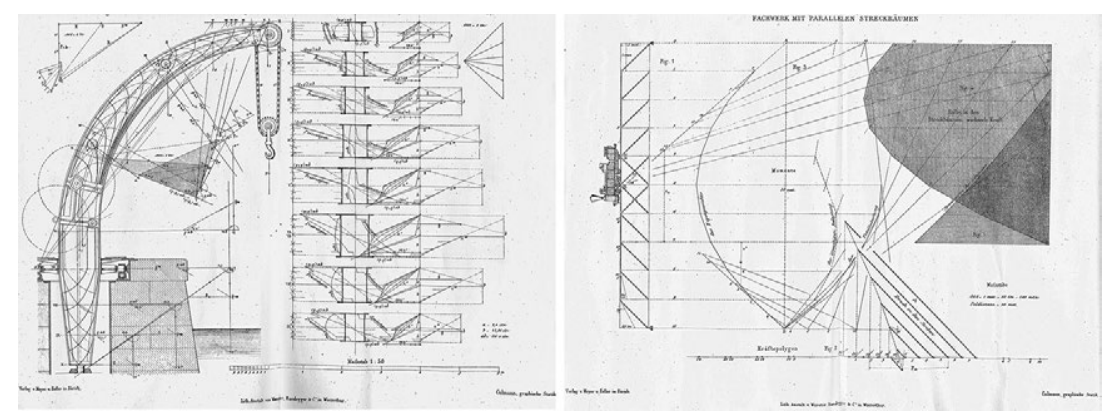

Fig. 5. Karl Culmann: Static graphics applied to engineering equilibrium problems: a crane, a railway bridge with a moving concentrated load [14].

The mathematician and politician Luigi Cremona (1830-1903) gave a significant contribution to graphical statics with Cremona's diagram, especially studying trusses (Fig. 6) [16].

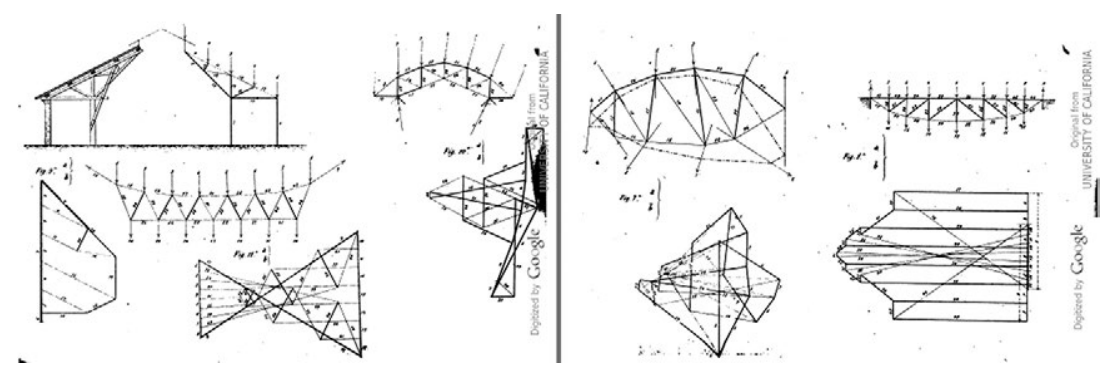

Fig. 6. Examples of Cremona's diagram [16].

\section{STABILITY}

So far we have seen a brief summary of the static equilibrium concept, defined as sum of forces (and angular momentum) equal to zero. However, sometimes equilibrium is precarious (Fig. 7) and at a certain point it seems to disappear. 

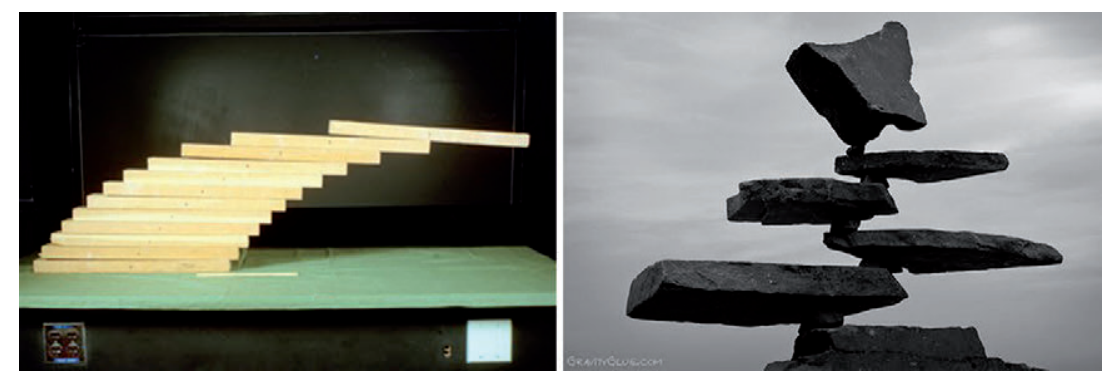

Fig. 7. Examples of precarious balance.

This happens, for instance, when a compressive load is applied to a sufficiently slender body, e.g. a ruler (Fig. 8). This is the famous Euler buckling problem (Fig. 9), first investigated and solved by Leonhard Euler (1707-1783), who was able to compute the force threshold, known as 'critical load', for the activation of this phenomenon. [17, 18].

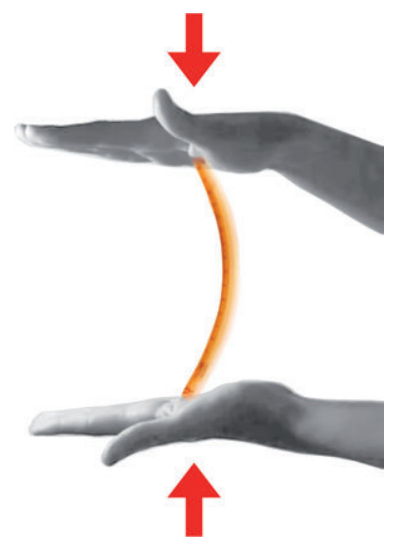

Fig. 8. Ruler subjected to compressive load.

A careful check shows that the straight equilibrium configuration always exists, for whatever value of the axial load (remaining in the elastic range). This can be easily understood by noting that in this configuration the sum of forces and angular momentum is trivially equal to zero, since they are always two identical and opposite forces acting on the same line. This is also confirmed mathematically by the picture of Fig. 10, known as bifurcation diagram, revealing that the rest configuration always exists. What happens at the critical threshold is (i) that two buckled configurations appear, one on the left and one on the right, 
and (ii) that the rest configuration is no longer visible in practice (while existing theoretically).

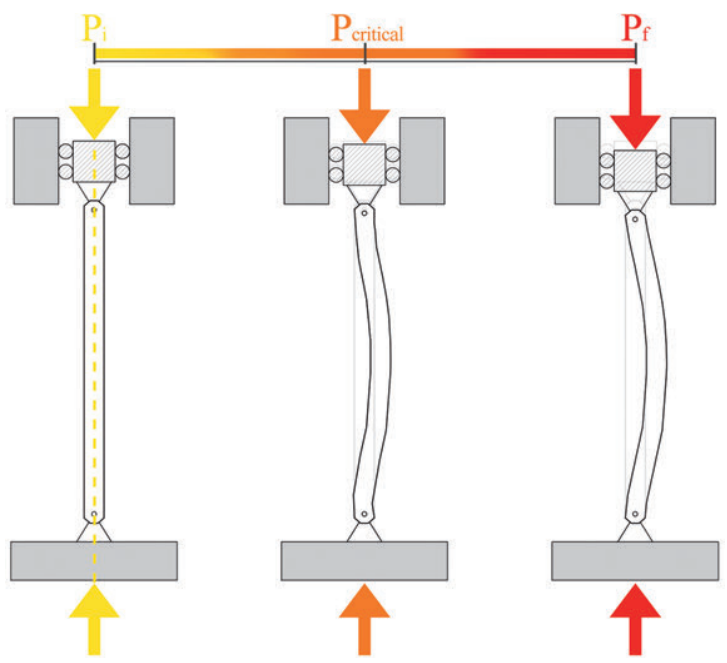

Fig. 9. Euler's critical load configurations:

Initial Load (Pi<Pcr), Critical Load (Pi=Pcr), Final Load $(P f>P c r)$.

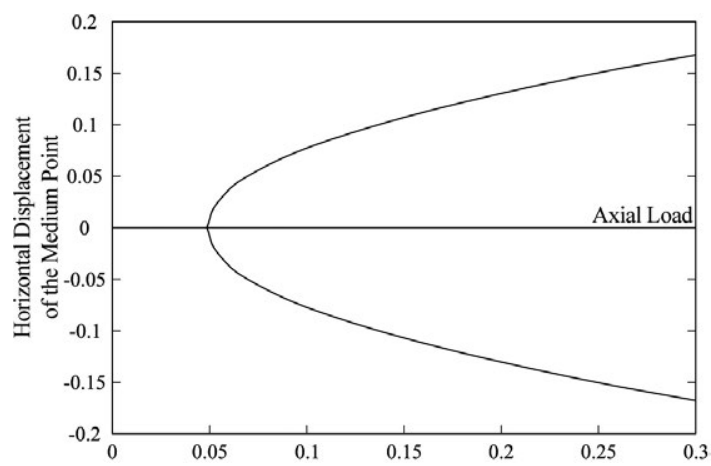

Fig. 10. Bifurcation point in the loading path.

The conclusion is that at the critical load we are not losing equilibrium, and something different happens.

It is the 'quality' of equilibrium that is lost; understanding this point was another major improvement in the knowledge of basic features of dynamics. 
A new concept was needed, that was named 'stability'. The intuitive idea of stability in the Lyapunov sense [19] (Aleksandr Michajlovi Lyapunov, 1857-1918) is that for 'small' perturbations from the equilibrium state at a certain initial time, subsequent motions should not be too 'large'. In other words, initial conditions close to an equilibrium solution tend to remain close along the time. Like the popular example of a ball at the bottom of the well. On the contrary, an equilibrium is unstable if small perturbations lead to motion that diverges from the equilibrium, as it happens to a ball on the top of a hill.

Stability is an inherently dynamic concept, but sometimes it can be treated as a static phenomenon for the solution of some important engineering problems. One of these is the aforementioned Euler's problem [17]: what characterizes the problem is a loss of stability (at the critical load), not equilibrium, without dynamic effects. Actually, it can be affirmed that Euler had already understood the concept of stability, although Lyapunov gave its exact definition long afterward.

Later on, mathematicians framed the Euler's problem and other similar problems that have been observed in the meantime by scientists and engineers in a unified viewpoint, and developed the 'theory of bifurcations' [20], in which the loss of stability was investigated systematically, and can be seen as a bifurcation point in a loading path. For smooth systems the obtained results look exhaustive, and only extremely pathological cases still need to be investigated [21], while for nonsmooth systems there is not yet a comprehensive classification [22, 23].

It is worth to remark that the theory of bifurcations is an inherently nonlinear topic.

One of the major results of the theory of bifurcations is that there are only few types of simple bifurcations of equilibrium points. In addition to the saddle-node, that will be discussed in the next section and that has different features, they are the supercritical pitchfork bifurcation (Fig. 11), where two buckled stable solutions exist above the bifurcation point (critical load); the subcritical pitchfork bifurcation (Fig. 12), where the buckled solutions are both unstable and exist below the bifurcation value; and the transcritical bifurcation (Fig. 13), where two equilibrium paths cross and exchange their stability.

All these cases have the common feature of having a stable equilibrium solution existing below the bifurcation point, where it continues to exist although losing stability. This is why they were often indistinct by engineers in the past, although the post-critical behavior is strongly different, both theoretically and practically. 

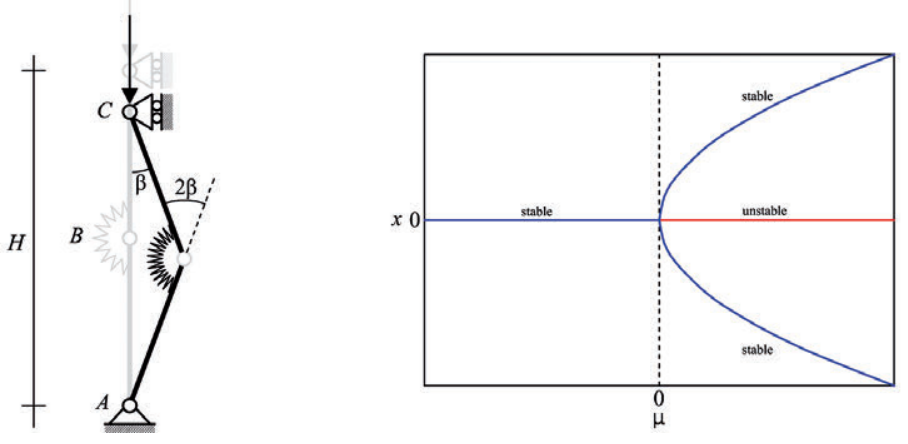

Fig. 11. Supercritical Bifurcation.
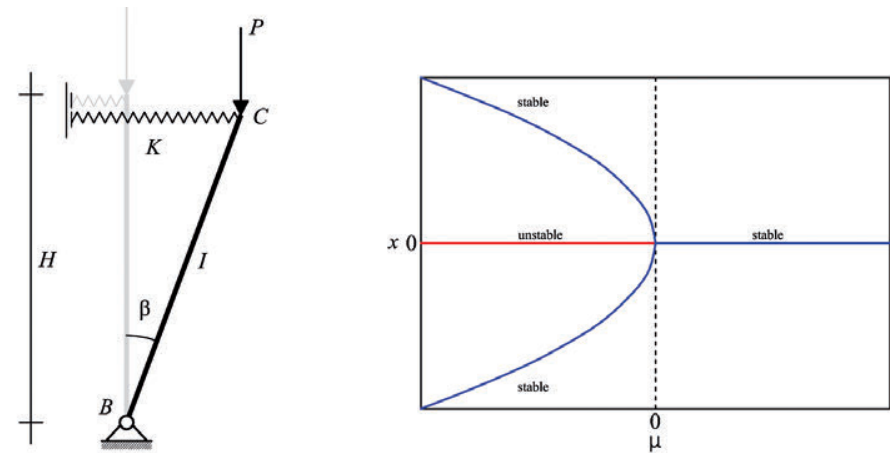

Fig. 12. Subcritical Bifurcation.
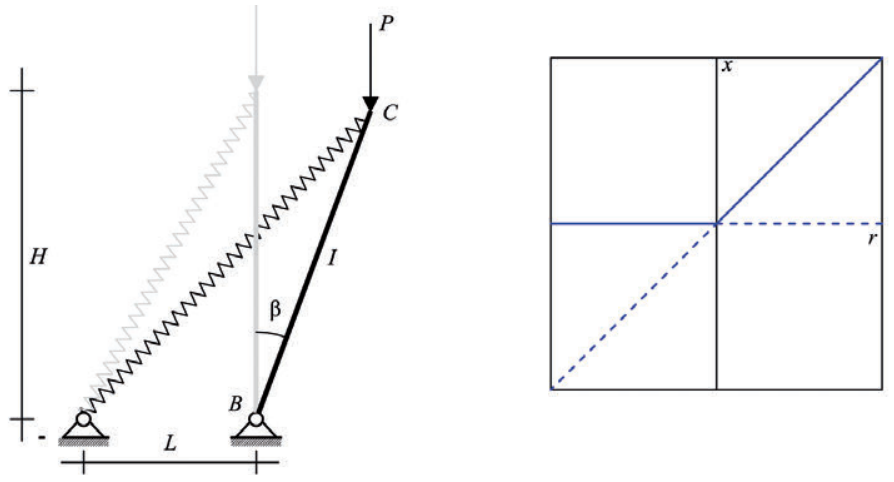

Fig. 13. Transcritical Bifurcation. 


\section{From LOSS OF STABILITY TO LOSS OF EQUILIBRIUM}

The above examples were engineering problems where the loss of stability does not cause a loss of equilibrium. However, a dynamical system can lose not only stability but also equilibrium for a varying configuration, or simply for a varying parameter. In the simplest way this happens when two equilibrium paths, one stable and one unstable, meet and coalesce for a varying parameter. This phenomenon, known as 'snap-through buckling' [24, 25, 26, 27] in engineering language, and saddle-node bifurcation [28] in mathematical language, implies that at the bifurcation point the system jumps instantaneously to another equilibrium configuration (or to another type of motion, indeed), existing elsewhere in the configuration space [29] (Fig. 14).

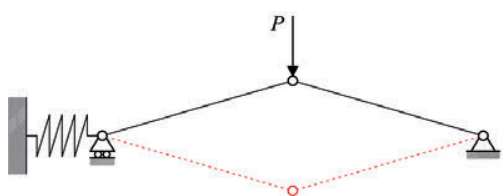

(a)

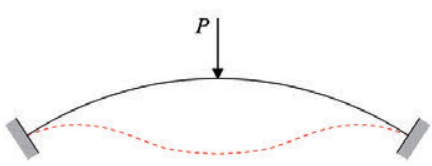

(b)

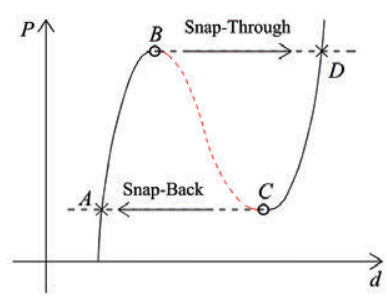

(c)

Fig. 14. Snap-through buckling: (a) initial and post-snap configurations of a SDOF arch (discrete system), (b) initial and post-snap configurations of a shallow arch (continuous system), (c) force-displacement curve.

To better understand this phenomenon, let us consider a tennis ball cut in two parts and suppose to take one of these. If we apply a compression load on the convex part of the ball, we can observe the destabilization process until the achievement of another stable equilibrium configuration (inverted configuration) (Fig. 15).

Snap-through buckling phenomena pose some of the most difficult problems in nonlinear structural analysis [24].

It is worth noting that the saddle-node bifurcation is the most frequent and probable in applications, while the other cases (pitchfork and transcritical) usually happen under particular conditions, such as the absence of imperfections or the presence of symmetries (see Sect. 8 for more details). In fact, it is possible to show that generic perturba- 
tions of the system transform a pitchfork or a transcritical bifurcation into a saddle-node bifurcation.

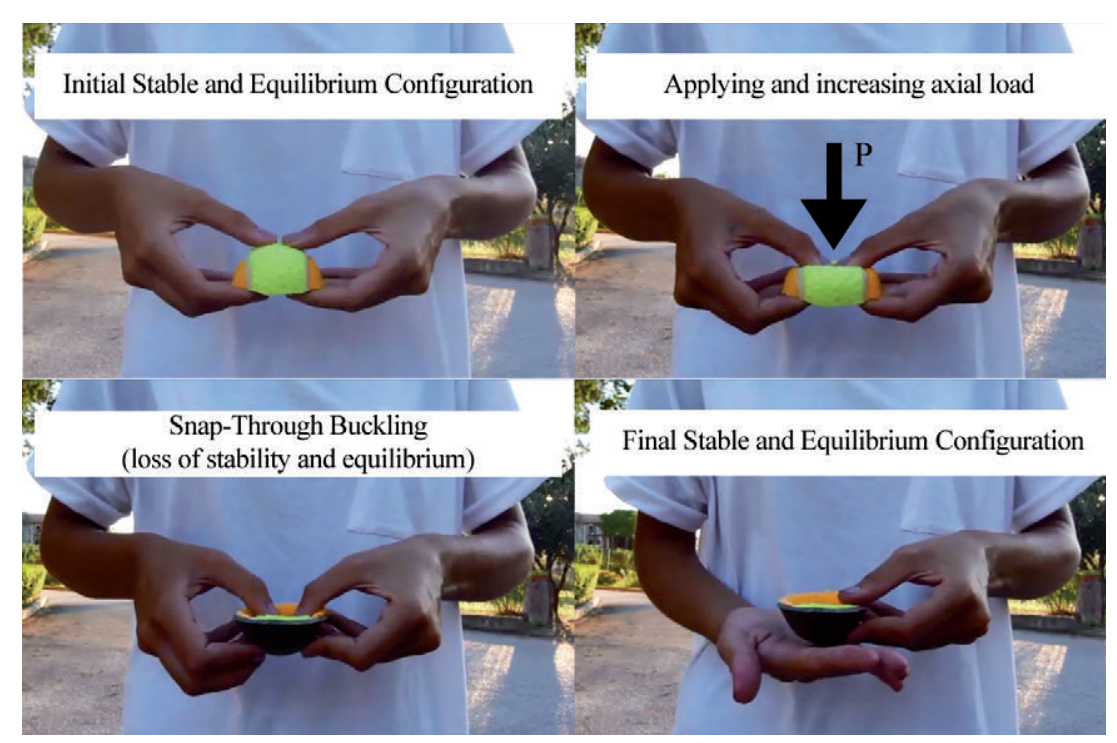

Fig. 15. Example of snap-through buckling: the tennis ball.

Although the saddle-node bifurcation represents the generic case from the dynamical point of view, it is not the best bifurcation for illustrative purposes. This because equilibrium and stability, which are different concepts, disappear simultaneosly, whereas in the other cases of bifurcation they are disjointed. This is likely the reason why the latter have been investigated first in the history (e.g. the Euler's problem), probably leading to the discovery of the stability concept.

What we have seen so far are local bifurcations, which deal with phenomena happening near an equilibrium point. It is possible to introduce a mathematical comprehensive bifurcations classification based on how the stability of an equilibrium solution is lost. To get this goal, let us consider the eigenvalues of the linearized system around the equilibrium position. If all the eigenvalues have a negative real part, the equilibrium is stable; if at least one eigenvalue has a positive real part, then it is unstable.

In this respect, the loss of stability can easily be seen as the crossing of the imaginary axes (the real part passes from negative to positive) by varying a governing parameter. 
The local bifurcations illustrated above correspond to a real eigenvalue becoming zero, plus some additional conditions for the transcritical and pitchfork bifurcations (and are just these extra conditions that make them uncommon). This provides a mathematical method to detect the bifurcation point.

The local stability, however, can also be lost when a couple of complex conjugate eigenvalues crosses the imaginary axis. This is an intrinsically dynamical phenomenon, known as Hopf bifurcation [30], that will be discussed in the following section.

\section{DYNAMICS}

The Hopf bifurcation corresponds to the local birth (or death) of a periodic solution from an equilibrium one as a certain parameter crosses a critical value. It is the simplest bifurcation not just involving equilibrium and, therefore, it belongs to what is sometimes called dynamic bifurcation theory [31]. In fact, the 'static approximation' which is valid for the previous bifurcations is no longer valid here. Dynamics eventually becomes the central topic.

As pitchfork, also Hopf bifurcation can be supercritical or subcritical, resulting in stable or unstable limit cycles ensuing from the equilibrium point, respectively. In particular, when a stable limit cycle surrounds an unstable equilibrium point, the bifurcation is supercritical. If the limit cycle is unstable and surrounds a stable equilibrium point, then the bifurcation is subcritical (Fig. 16).

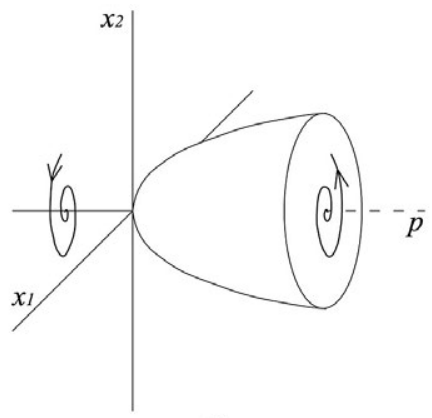

(a)

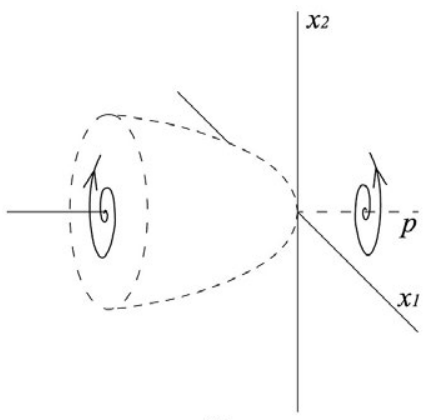

(b)

Fig. 16. The Hopf Bifurcations: (a) supercritical, (b) subcritical. 
Likely, the most famous realization of the Hopf bifurcation in the real world is the phenomenon known as flutter. It can be encountered in flexible structures subjected to aerodynamic forces, such as aircrafts, buildings and bridges. Flutter occurs as a result of interactions between aerodynamics, stiffness, and inertial forces on a structure. When the speed of an aircraft increases, there may be a point at which the structural damping is insufficient to damp out the growing motions due to aerodynamic energy. In the worst case this vibration can lead to structural failure $[32,33]$. Other meaningful examples concern bridges. In particular, from the famous case of the Tacoma Narrows Bridge (Fig. 17), it can be observed that due to the aeroelastic fluttering the structure started to oscillate with an extremely large amplitude, that eventually leads to the collapse.
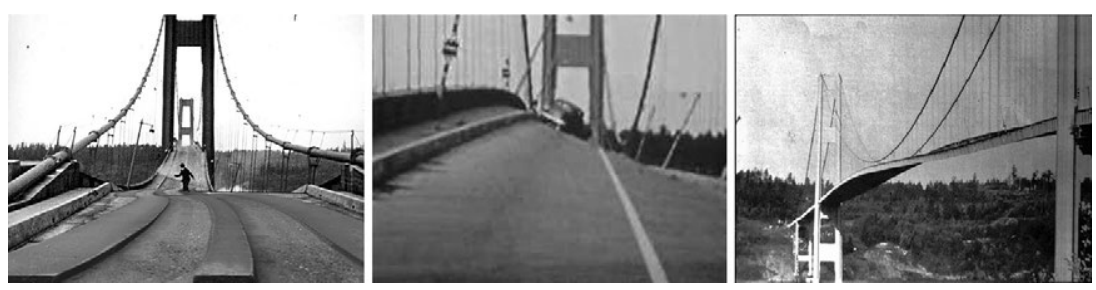

Fig. 17. Fluttering phenomenon: the case of the Tacoma Bridge.

Another practical realization of Hopf bifurcation is known as galloping, and happens in frozen telecommunication wires. The ice around the wire changes the geometrical cross-section and its aerodynamic properties, so that the wind can active oscillations even for small values of its velocity. The mechanism is very similar to flutter, although they have been named differently, likely as a consequence of the fact that in flutter the aerodynamic coupling occurs with the 'original', while in galloping it occurs with a 'modified' (by the surrounding ice) structure.

Finally, a further common life example of Hopf bifurcation occurs in garden hoses: when the velocity of the water overcomes a certain threshold, the hose starts to oscillate.

Note that in all the previous examples the governing parameter leading to the Hopf bifurcation is the velocity of the gas/air/water.

The Hopf bifurcation is not the only dynamical phenomenon that attracted the attention of researchers in the past. Another one is 'resonance', that entails large oscillations of the system as a consequence of 
small excitations (Fig. 18) [34]. This amplification is obtained when the period of the excitation is close to a natural period of the structure, which is an intrinsic property of dynamical systems, just like the tone of the voice is a characteristic of each person. The frequency at which the amplitude of oscillation becomes maximum is called resonant frequency.

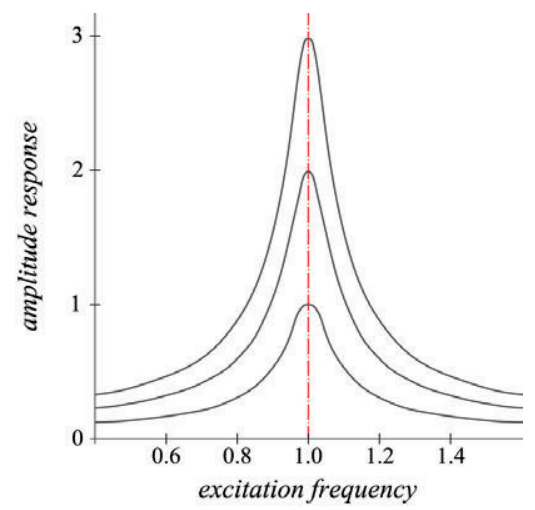

Fig. 18. The amplification of the system response due to resonance.

A practical realization of this phenomenon occurs in common swings. In this case, children deliberately look for amplification of the oscillations, and exploit this phenomenon by properly tuning the external (provided by the parent that pushes, Fig. 19a) or the parametric (provided by themselves, Fig. 19b) excitation. In fact, with relative small pushes, but at the right time interval, it is possible to obtain large displacements.

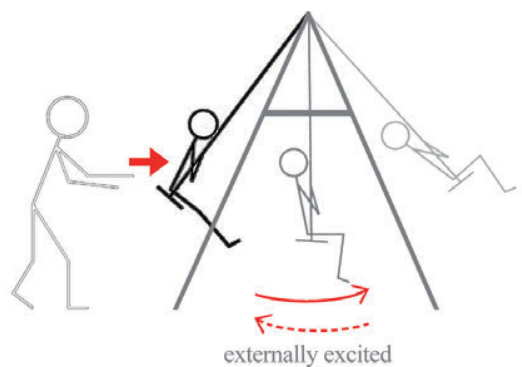

(a)

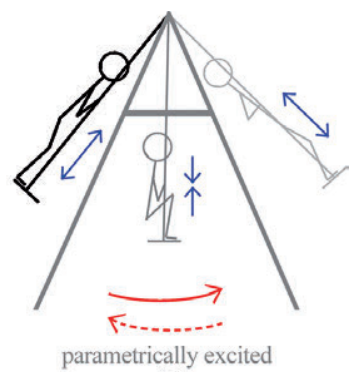

(b)

Fig. 19. Resonance in the case of playground swings:

a) externally excited, b) parametrically excited. 
On a more serious side, resonance can be observed also on structures subjected to an earthquake, in many mechanical engineering applications, and it is responsible for other curious phenomena, such as the breaking of a glass when someone screams at the 'right' frequency (resonance frequency).

When resonance occurs in nonlinear systems, it is called nonlinear resonance and it has some specific characteristics. For example, the resonant frequency is no longer an intrinsic characteristic of the system (as occurs for liner systems), but it depends on the amplitude of the excitation. In this respect, it can be affirmed that Galileo was wrong on pendulum isochronism for high amplitudes, while being right for small ones. Nonlinear effects may significantly modify the shape of the resonance curves, bending them toward low frequencies (softening behavior, Fig. 20a) when the nonlinear term decreases the linear stiffness, or toward high frequencies (hardening behavior, Fig. 20b) in the opposite case [35].

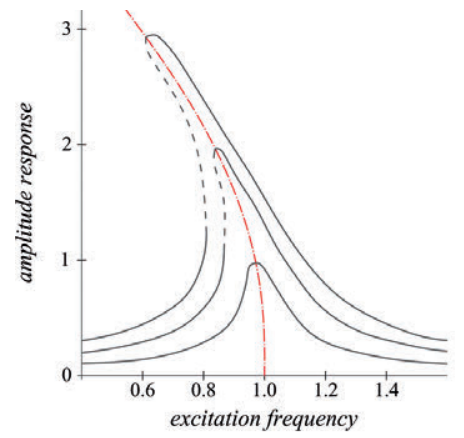

(a)

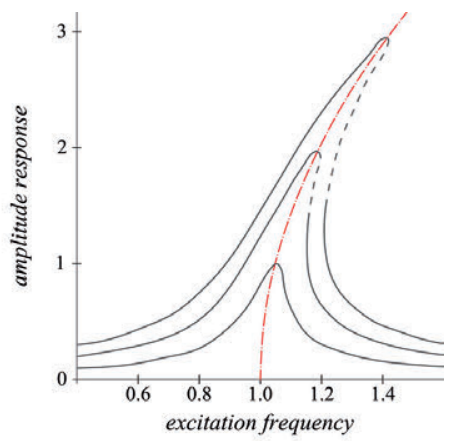

(b)

Fig. 20. Nonlinear resonance curve response: (a) softening behavior, (b) bardening behavior.

The nonlinear behavior produces also other interesting phenomena, known as sub- and super-harmonic resonances, where the amplification occurs if the external excitation is an integer multiple (or submultiple) of the natural frequency.

Furthermore, in the nonlinear resonance curves some local bifurcations can appear for large excitation amplitudes, leading to the jump phenomenon and to the nonlinear hysteresis that is obtained increasing and then decreasing the excitation frequency. This eventually links bifurcation and resonance. 
Finally, also internal resonance may occur. It happens when the dynamical system has two different natural frequencies that are one an integer multiple of the other. It entails the exchange of energy between different vibrations modes of the structure, which can be dangerous if not properly taken into account.

\section{BIFURCATIONS OF PERIODIC ORBITS}

As explained above, through the Hopf bifurcation it is possible to have periodic motions from equilibrium solutions. But periodic orbits can exist independently of being born from an Hopf bifurcation, and are instead the common response as a consequence of periodic excitations.

Thus, the stability problem concerns also periodic solutions, and the Lyapunov definition of stability, properly reformulated, applies.

The bifurcation theory has been developed for this kind of motion $[36,37]$, too, and it was found that also in this case the local stability is governed by the eigenvalues of an appropriate matrix describing the local behavior around the periodic solution after one period. According to the Floquet theory [38], the periodic solution is stable if all the eigenvalues have a modulus smaller than 1 , unstable if at least one eigenvalue has a modulus larger than 1 . It is then clear that the loss of stability (bifurcation) occurs when, varying the governing parameter, one eigenvalue gets modulus equal to 1 .

In non-pathological cases, this can happen in three different way. The first is when a real eigenvalue becomes equal to 1 . The associate bifurcation is called saddle-node (for periodic orbit), and it is very similar to the corresponding one for equilibrium points: one stable periodic orbit and one unstable periodic orbit coalesce and jointly disappear.

The second case is when a real eigenvalue becomes equal to - 1 . It has no counterpart for equilibrium points and it is named period-doubling bifurcation because after the bifurcation the periodic orbit survives but becomes unstable, and another stable periodic orbit appears with a period twice that of the original orbit.

Finally, the last case corresponds to a couple of complex conjugate eigenvalues having modulus exactly equal to 1 . This somehow corresponds to the Hopf bifurcation for equilibrium points, and is named secondary Hopf or, better, Neimark-Sacker bifurcation. Here the periodic orbit survives but loses stability, and a stable quasi-periodic solu- 
tion appears. A quasi-periodic solution is an oscillation which has two periods, incommensurable between each other, like for example $c_{1} \sin \left(\omega_{1} t\right)+c_{2} \sin \left(\omega_{2} t\right), \omega_{1} / \omega_{1} \in \mathbb{R} \backslash \mathbb{Q}$.

As for equilibrium solutions, there are also uncommon bifurcations, similar to the transcritical and pitchfork ones, that require some extra conditions. They will not be discussed here.

Practical examples of Neimark-Sacker bifurcations are the shimmy and wobbling phenomena, although of course many other real world examples exist. In aircrafts, shimmy is an oscillatory, combined lateral-yaw motion of the landing gear caused by the interaction between dynamic tire behavior and landing gear structural dynamics (Fig. 21).
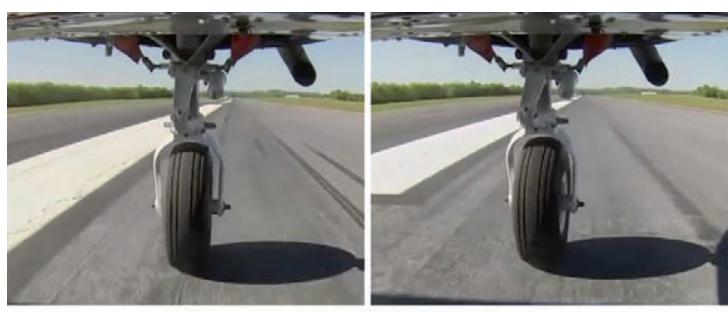

(a)

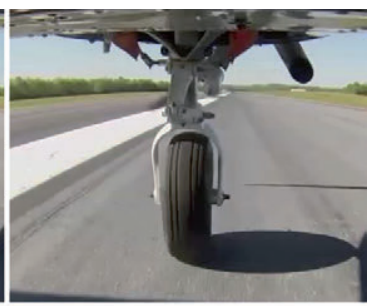

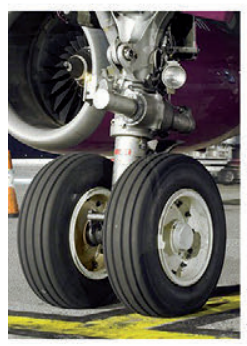

(b)

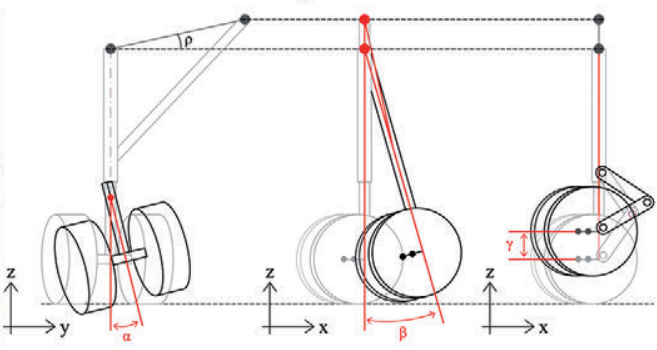

(c)

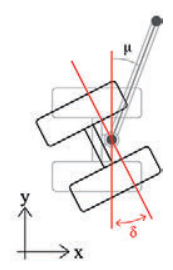

Fig. 21. (a) Shimmy of the main landing gear, (b) Examples of aircraft dual-wheel main
landing gear, (c) dynamic behaviors of the tires and the dual-wheel gear structure.

The amplitude of the motion caused by shimmy may grow up to a level of annoying vibrations affecting the comfort and visibility of the pilot, or can even result in severe structural damage and landing gear collapse [39].

Shimmy not only occurs on aircrafts but has also been encountered on the steerable wheels of cars and motorcycles. In the latter case 
this phenomenon is called wobbling and can be defined as an unstable, dangerous, oscillation of the front tire around steering axis (Fig. 22).

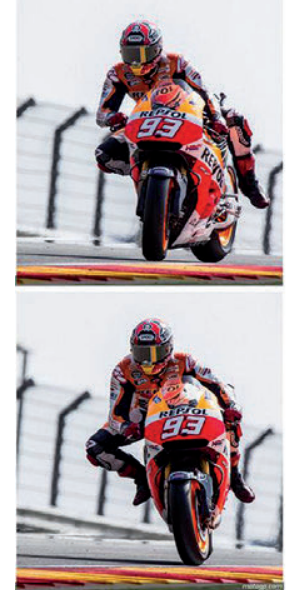

(a)

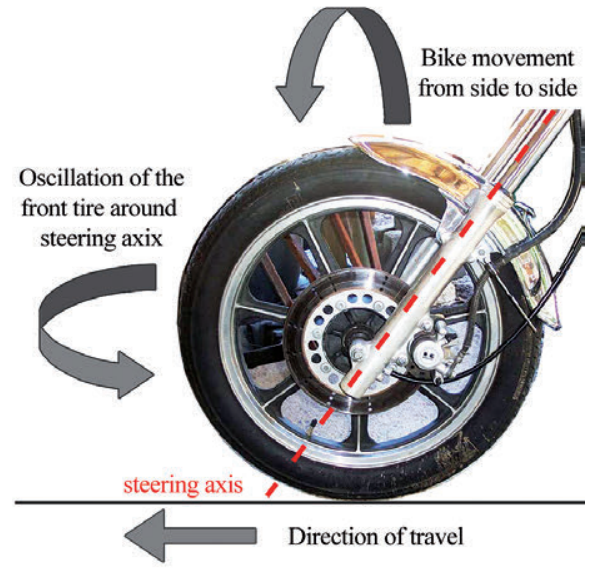

(b)

Fig. 22. The Wobbling phenomenon: (a) example of wobble, (b) dynamic of the front tire.

\section{OTHER KIND OF MOTIONS AND OTHER KIND OF BIFURCATIONS}

In the previous sections it has been shown that three types of motion may exist: equilibrium, periodic and quasi-periodic solutions. One would wonder if this list is exhaustive, or whether there are other types of motion. Certainly it is not difficult to imagine quasi-periodic solutions having a large number of incommensurable frequencies. But this is not enough, since other kind of motion exist, named homoclinic and heteroclinic solutions. A homoclinic orbit is a trajectory of the dynamical system that connects an equilibrium point (a saddle, to be precise) to itself (Fig. 23a). A heteroclinic orbit, instead, is a trajectory that connects two different equilibrium points [28] (Fig. 23b).

Both are generally unstable, although they play a major role, in the background, in the organization of the dynamics of the system.

Finally, there is another kind of solution, lastly discovered, that it is necessary to mention: chaos. Chaotic motions are characterized by not having any period, never repeating forward in time although coming back close enough to any previous point, possibly by waiting for a long 
time interval. When chaotic motion is stable it is named chaotic attractor. An important property of chaos, related to its fractal nature, is its strong dependence on initial conditions: two extremely close starting points exponentially diverge forward in time along the attractor (Fig. 24). This means that, in chaotic regime, it is impossible to predict systems dynamic behavior long after the starting point (Butterfly effect) [40, 41], since in real world it is not possible to know exactly the initial conditions.

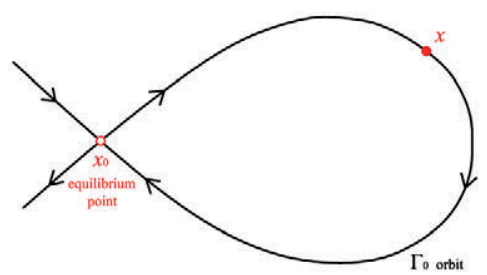

(a)

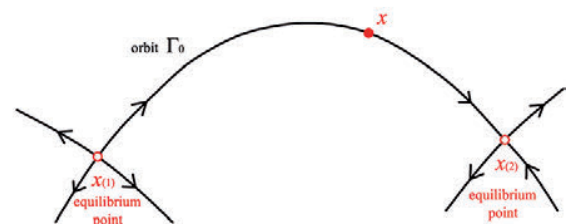

(b)

Fig. 23. (a) Homoclinic and (b) beteroclinic orbits on the plane.

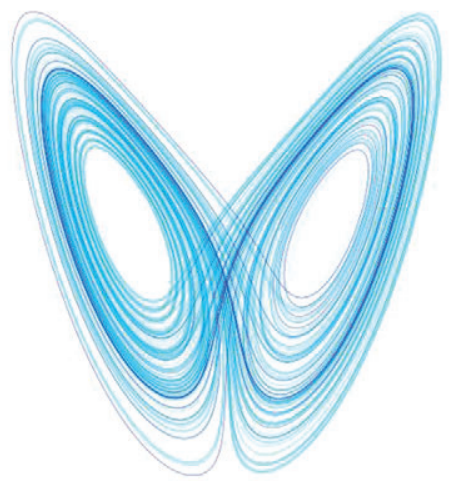

Fig. 24. Lorenz's strange chaotic attractor.

As far as bifurcations are concerned, we have seen so far: saddlenode, pitchfork (supercritical or subcritical), transcritical, and Hopf bifurcations when equilibrium solutions are taken into account; whereas saddle-node, periodic-doubling, and Neimark-Sacker bifurcations are those related to periodic solutions. Again, this list is not exhaustive, since other kind of bifurcations have been discovered theoretically, and sometimes observed (directly or indirectly) in practice. They are the degenerate local bifurcations (in addition to transcritical and pitchfork), bifurcations of non-smooth systems (which are plenty of new and unexpected behaviors), and global bifurcations, such as homoclinic and heteroclinic 
bifurcations, that have the characteristic of involving the 'whole' dynamics of the system and not a specific orbit. Global bifurcations often occur when some invariant sets, commonly stable or unstable manifolds, collide between each other, causing changes in the topology of the trajectories in the 'whole' phase space. They can also involve chaotic attractors [41], and in this case sometimes they have been called crisis [42].

\section{EFFECT OF IMPERFECTIONS IN THE SYSTEM}

In the previous sections we have seen how stability influences the quality of the solutions, determining, in brief, if we can see them in real world or not. Stability is concerned with imperfections in initial conditions.

We have not considered so far imperfections in the dynamical system itself, which can be different from the expected or designed, due to many reasons, including uncertainties in the production processes. This can be mathematically translated as uncertainties on the system parameters, which can be different from the nominal ones.

Warner T. Koiter (1914-1997) [43, 44] was the first to understand that structural imperfections can significantly influence the load bearing capacity, causing its strong decrement. Because of imperfections, structures can also lose stability (or equilibrium) for a value of the governing parameter much different from the theoretical one (Fig. 25) [45].
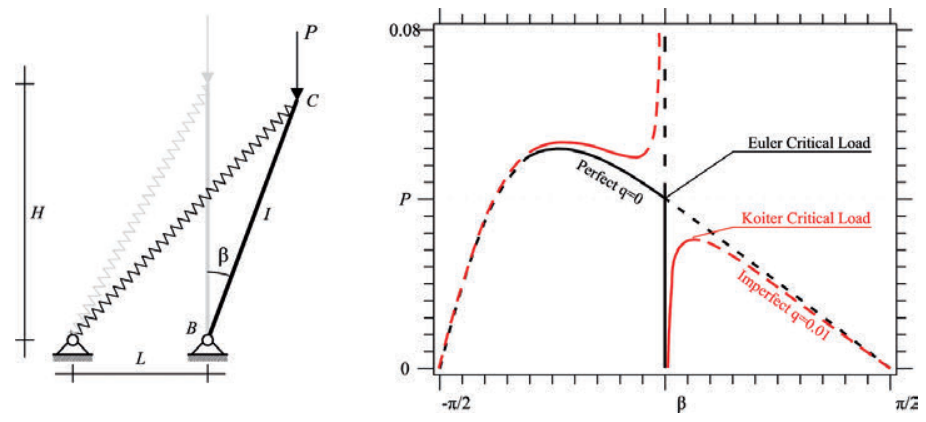

Fig. 25. Comparison between Euler and Koiter critical loads.

Studying the effects of the system imperfections on the system response is known as 'structural stability theory' and, although being initially developed independently, it is nowadays a branch of the bifur- 
cation theory, which gave a mathematical unified framework to the engineering intuitions. The novel idea of the structural stability theory is to consider perturbations of the system instead of the perturbations of initial conditions typical of the classical (Lyapunov) stability theory.

Within structural stability, a bifurcation is named 'structurally stable' if small perturbations in the parameters do not modify the qualitative features of the bifurcation, whereas 'structurally unstable' stands for the opposit case. In this respect, and accordingly to what we have seen before, saddle-node and Hopf are structurally stable bifurcations of equilibrium points, while transcritical and pitchfork are structurally unstable; saddle-node, period-doubling and Neimark-Sacker are structurally stable bifurcations of periodic orbits.

Note that in structurally unstable bifurcations commonly there is not only a qualitative change of the bifurcation (e.g. from pitchfork to saddle-node), but also the quantitative threshold for bifurcation changes dramatically by adding of system imperfections. It is just this kind of problems, applied to buckling of shell, that triggered the Koiter studies.

A significant example that demonstrates the influence of imperfections on the load carrying capacity is represented by thin shell structures, such as tanks [46]. In addition to practical occurrence of the phenomenon (Fig. 26a), this can also be seen when these kind of structures are analyzed, for example by the finite element method (Fig. 26b), within a nonlinear framework. In this case the artificial imperfection introduced by the discretization adds up to the physical imperfections, and commonly helps in detecting the reduction of the load carrying capacity. The sensitivity to imperfections is shown in Fig. 26c, where the buckling load of a geometrically perfect shell is compared with a shell accounting for geometric imperfections. As it may be seen, there is a considerable reduction in buckling load with increased imperfection [47].

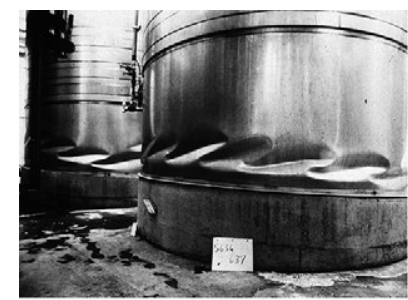

(a)

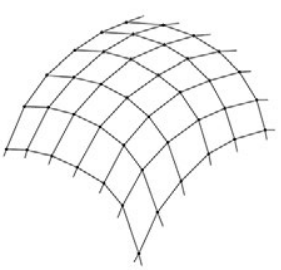

(b)

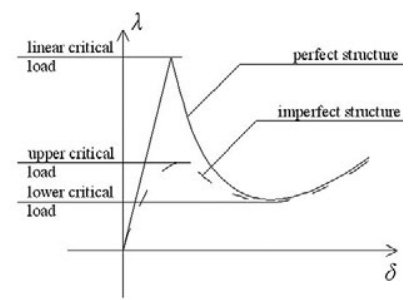

(c)

Fig. 26. Example of thin shell structures: (a) non-symmetrically buckled tank [46],

(b) geometric imperfections introduced by faceted geometry of typical finite element meshes, (c) comparison between perfect and imperfect shell load-deflection curves. 


\section{DyNAmical INTEGRITY}

In the 90s, J. Michael T. Thompson (1937-) $[48,49,50]$ has reconsidered the classical Lyapunov stability concept. He started from observing that in the classical definition of stability the considered perturbations are infinitesimal, while in the real world even the smallest perturbations have a finite amplitude. Thus, according to Thompson, in the real world a system should be able to accommodate finite, although small, changes in the initial conditions, in order to actually experience the considered solution. Otherwise, it may happen that the considered solution cannot be seen in practice, even if it is theoretically stable - with however only infinitesimally small perturbations of initial conditions allowed. He moved from 'theoretical' stability to 'practical' stability, and sometimes the word 'robustness' is used to refer to the latter case.

Although this basic idea is simple, its practical implementation is far from trivial, and gives rise to the research field today known as dynamical integrity [53].

It is worth noting that Koiter refers to perturbations of the structure (geometry, materials, etc.), and thus operates in the field of structural stability (with major effects even in the static case), while Thompson, like Lyapunov, refers to perturbations of initial conditions, and contributes to the theory of (nonlinear) dynamical systems [51].

Thompson's idea can be reformulated by saying that "if the basin of attraction is not large and compact enough, the solutions cannot be seen in the real world" [52]. In order to better understand this concept, it is necessary to define the basin of attraction. A basin is a subset of the phase space made of initial conditions sharing a common property. If this property consists of having the same attractor, i.e. converging forward in time to the same solution, this set is named basins of attraction.

In this context, dynamical integrity consists of the systematic study of topology, evolution, changes by varying parameters, etc. of basins of attraction. They can be very complex (even fractal), so that dynamical integrity is not the simple measure of the basin magnitude.

To fix ideas, look at Fig. 27. It represents the same system (a Duffing equation) at different values of the excitation amplitude, leading to different basins of attraction. The first one (Fig. 27a) has a large compact part of the basin (see for example the area within the circle) and thus it can be safely used in practice. The second one (Fig. 27b), on the other hand, has a very small compact part of the basin of attraction 
(again, see the area within the circle) and thus it is totally unsafe in practice, even if it is stable according to Lyapunov.

To underline that dynamical integrity is not simply the study of the magnitude of the basins of attraction, we remark that the whole area (not only the compact part) of the basin of the case of Fig. 27b is larger than the whole area of the basin of Fig. 27a. It is however spread in the fractal zone, and thus it is useless from a safety point of view. If we consider only the area, we can arrive to the wrong and unsafe conclusion that the situation of Fig. $27 a$ is better than that of Fig. 27b. Fractality makes the difference.

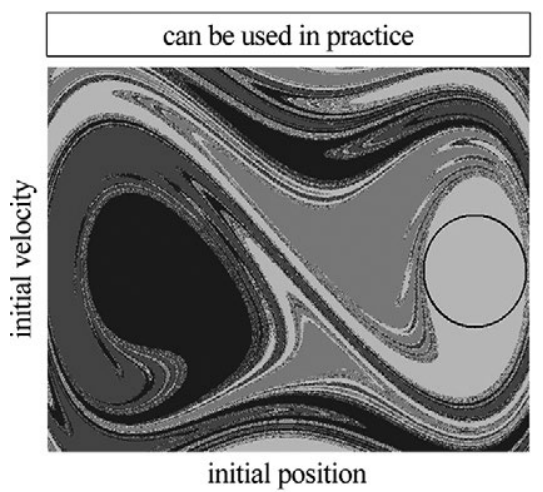

(a)

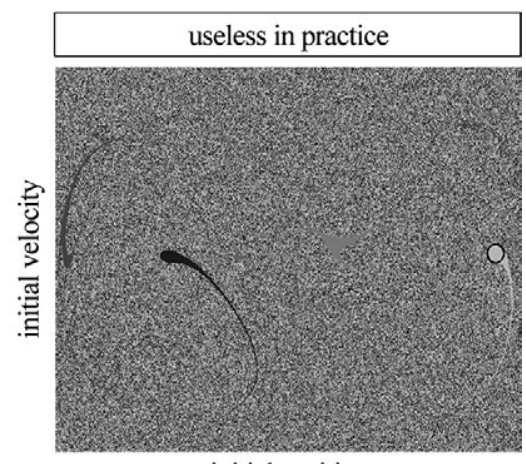

initial position

(b)

Fig. 27. Stable attractors with different basins of attraction:

(a) useful in practice and (b) useless in practice.

While stability is a local property of the attractor because it considers only what happens around the reference solution, dynamical integrity is a global property. In fact, to discuss the dynamical integrity it is necessary to study the basins of attraction, that spread around the whole phase space, and not only in a neighborhood of the attractor. Changing the paradigm from local to global dynamics means, from the one hand, to obtain more information, deeper knowledge of systems, wider use of results, but, from the other hand, to deal with growing difficulties in terms of numerical costs of the simulations.

First experimental confirmations of the usefulness of dynamical integrity concepts come from two practical applications, one at the macro scale (a rotating parametrically excited pendulum) and the other at the micro-scale (a Micro-Electro-Mechanical-System - MEMS), respectively [51]. 
The rotating pendulum, parametrically excited by the vertical motion of the pivot, is the orange rod in Fig. 28.

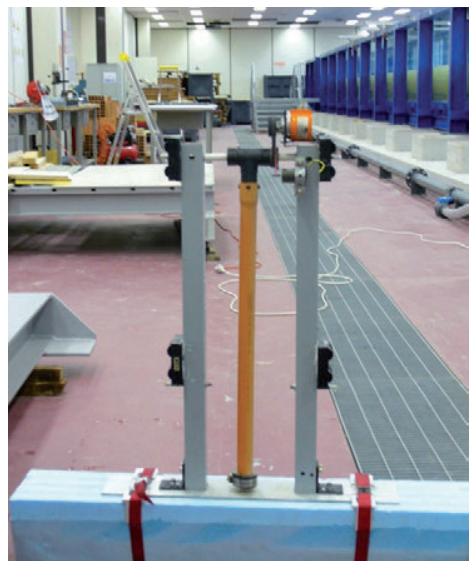

Fig. 28. The experimental rotating pendulum [51].

Rotating solutions, rather than oscillations, are considered because they are more interesting from a dynamical integrity point of view. This because they are less robust and, as a consequence, more affected by perturbations. They have small basins with respect to the competing oscillations, so that detecting them experimentally is challenging.

Fig. $29 a$ shows the region where rotations are theoretically stable (in the Lyapunov sense). Fig. 29b, on the other hand, shows that experimentally the rotations can be detected only along a central (w.r.t. the excitation amplitude $p$ ) and finite-magnitude strip, shrinking for low values of circular frequencies $\omega$. This strip, as it can be easily observed, is much narrower than the region of theoretical existence. The difference between the experimental and theoretical regions is too large to be attributed only to experimental uncertainties.

The results reported in Fig. 30 provide a clear justification of this difference. In fact, it is possible to see (Fig. 30a) that the dynamical integrity is large, according to a certain measure [57], only in a specific interval of increasing excitation amplitude (for fixed values of the excitation frequency), much smaller than the stability interval, which corresponds to a relatively robust attractor with a compact and not eroded basin. It is just in this interval that we observe rotations experimentally. This property holds for all frequencies (Fig. 30b), and it is a proof of the 
fact that in practice stable solutions with small basins of attraction cannot be obtained, which is the keystone of dynamical integrity.

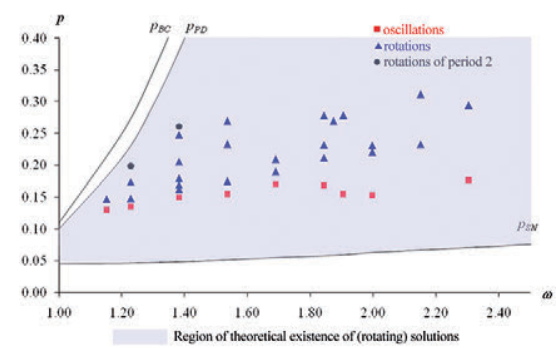

(a)

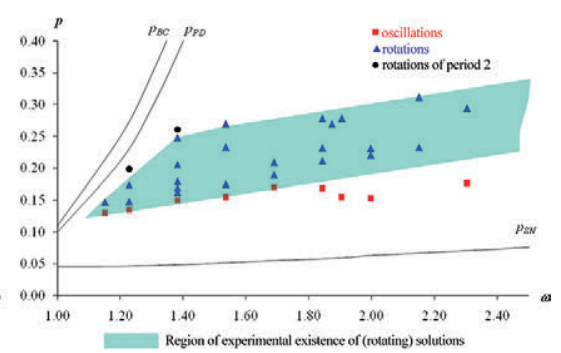

(b)

Fig. 29. The behavior chart in the ( $\omega, p)$ parameter plane: (a) Region of theoretical existence of solutions, (b) Region of experimental existence of solutions [51].

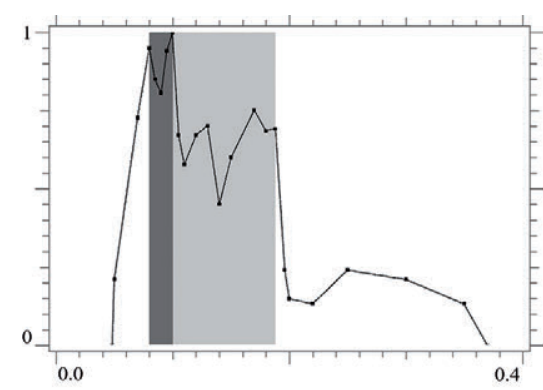

(a)

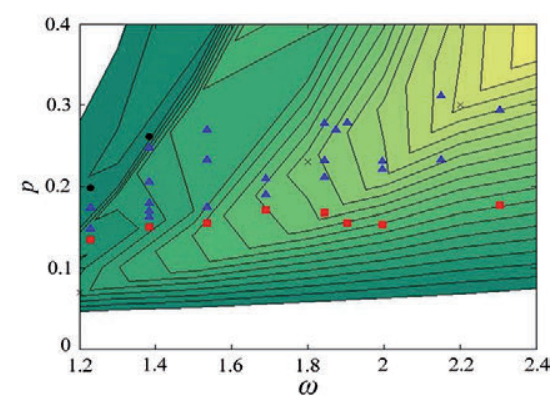

(b)

Fig. 30. (a) The integrity measure as a function of the amplitude $p$, for a fixed value of the forcing frequency $\omega=1.3$. (b) Contour plot of the integrity measure and the experimental data [51].

The second experimental verification of usefulness of dynamical integrity has been obtained with a MEMS-based capacitive accelerometer, consisting of a proof mass (upper electrode) suspended over a substrate (lower electrode) by two cantilever beams (Fig. 31) [54, 55]. The experimental setup is shown in Fig. 32 [54, 55, 56].

The frequency response of the accelerometer for a wide range of frequency $(1-400 \mathrm{~Hz})$ is reported in Fig. 33, which shows that around the resonant frequency there is an interval of frequencies where there are no bounded solutions. Here the proof mass sticks on the lower electrode, and the well-known dynamic pull-in phenomenon occurs, which 
is unwanted in applications [57]. From a mathematical point of view this phenomenon is named 'escape' since entails escaping from the potential well and approaching another attractor.

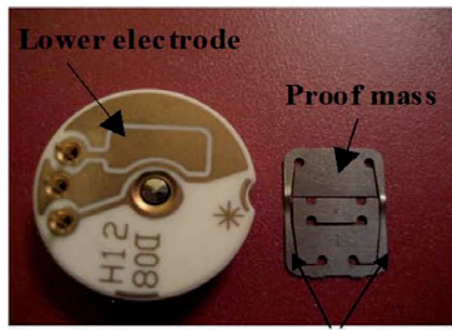

Cantilever beams

(a)

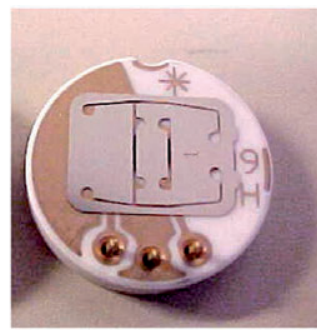

(b)

Fig. 31. The MEMS capacitive accelerometer, fabricated by Sensata Technologies and tested in [55]. (a) The device taken-apart; (b) The device assembled.

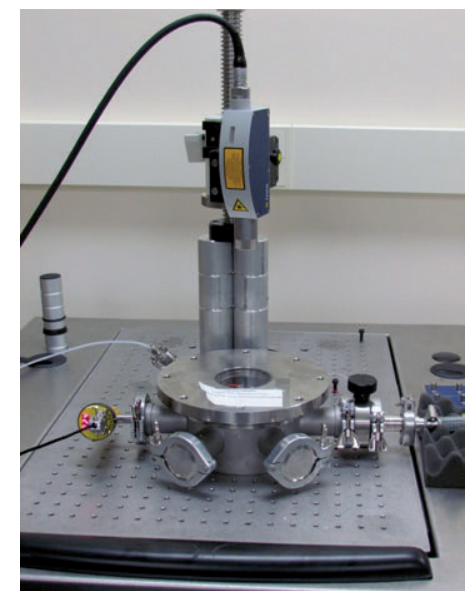

Fig. 32. Experimental test setup [55].

Building the frequency response curves for many values of the frequency allows to identify in the parameters space where pull-in occurs experimentally (Fig. 34).

As done before with the rotating pendulum, the experimental data are compared with the theoretical predictions of the pull-in region (Fig. 34). Theoretical pull-in is systematically above the experimental 
pull-in threshold, and a significant difference is observed. Again, this difference is too large to be ascribed only to the experimental uncertainties, although these are surely present, up to a certain extent.

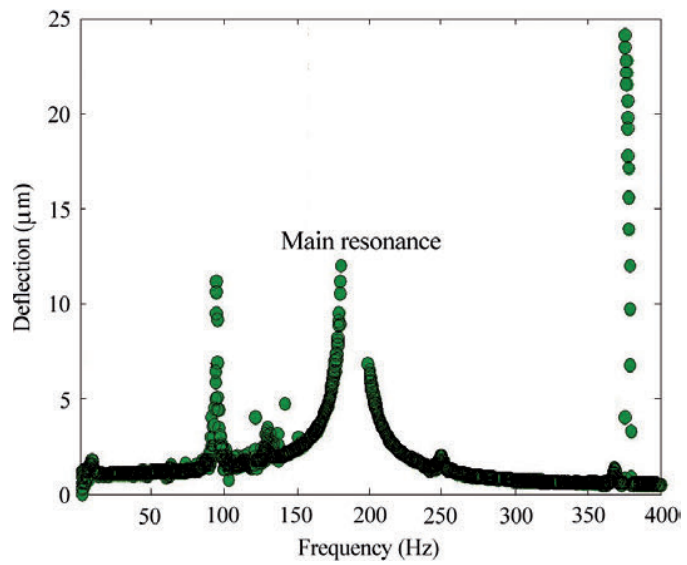

Fig. 33. A forward frequency sweep response for the capacitive accelerometer tested.

The figure shows three distinctive resonances: super-harmonics, sub-harmonic and primary resonance.

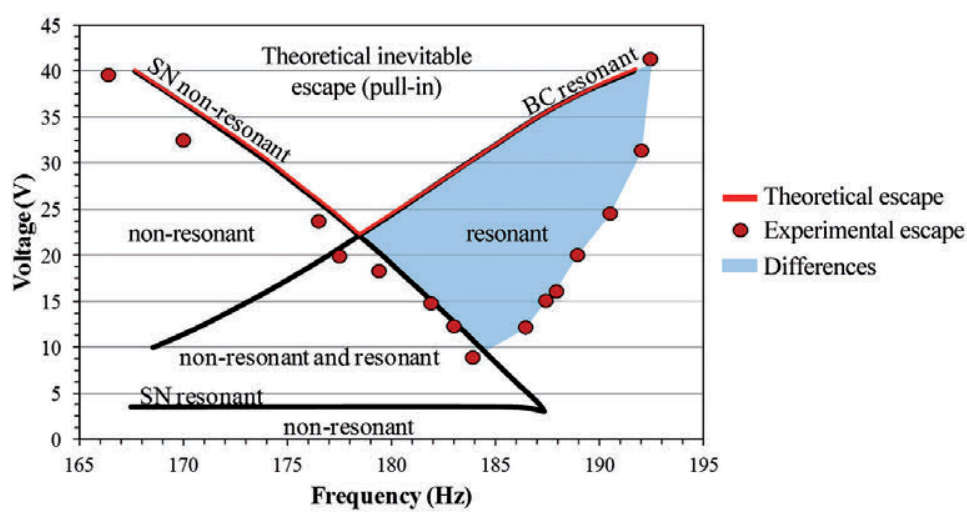

Fig. 34. Comparison between theoretical and experimental escape [51].

Also in this case the discrepancy can be explained by dynamical integrity. In fact, by determining the contour plot of the dynamical integrity measure for the bounded attractors (Fig. 35), it is possible to see that the experimental threshold of pull-in approximately stands 
along a level curve of the dynamical integrity measure. This shows that in practice the attractors disappear when the basins of attraction are no longer large and compact enough, even if the solution is theoretically stable, according to the dynamical integrity basic principle.

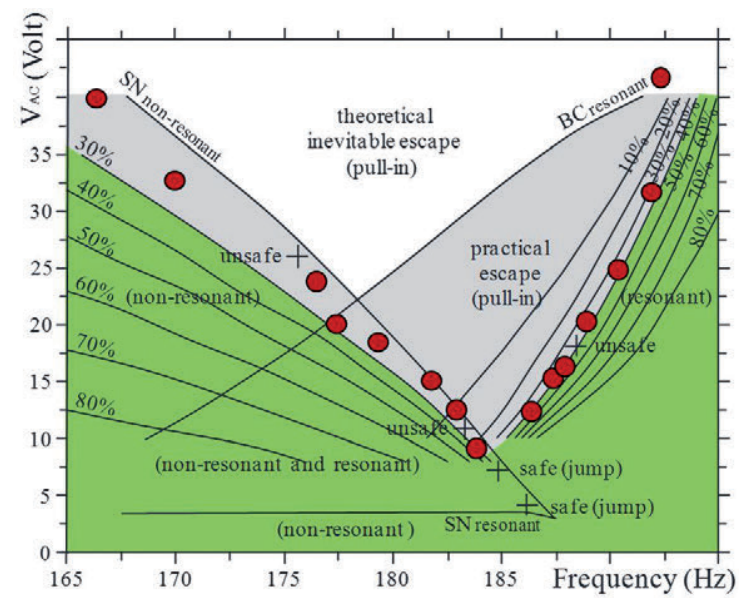

Fig. 35. Level curves of the dynamical integrity measure (Integrity Factor [51]).

\section{A CHALLENGE FOR THE FUTURE}

By means of the dynamical integrity it is possible to fully understand why and when certain theoretical solutions can be seen in real world, and when they are safe: the integrity measure must be large enough.

The basic tools for the dynamical integrity are the basins of attraction, that are very demanding from a computational point of view, as they are basically computed by brute-force like algorithms. This is the reason behind the fact that to date they have been built only for low dimensional systems, mainly those having one mechanical degree of freedom only.

But in real world dynamical systems have many degrees of freedom, up to infinity for continuous systems, and this calls for the necessity of having basins of attraction for large dimensional systems in order to investigate dynamical integrity of complex structures. This represents a challenge for the future, and some initial results have yet been 
obtained (Fig. 36) exploiting high performance computing, such as parallel computing [59].
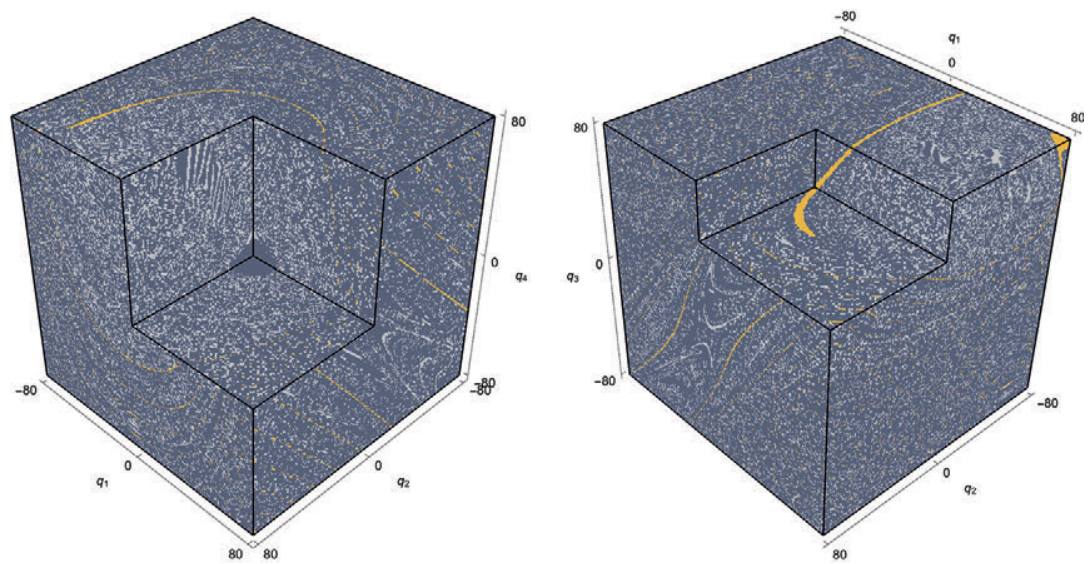

Fig. 36. Basins of attraction a couple of periodically forced Duffing oscillators.

\section{ACKNOWLEDGEMENT}

The authors wish to thank Prof. G. Rega, Prof. M. Younis, Dr. P. Belardinelli and Dr. L. Ruzziconi that collaborate to obtain some results summarized in this review paper.

\section{REFERENCES}

1. I. Newton, The Principia: Mathematical Principles of Natural Philosophy, translated by I. Bernard Cohen and Anne Whitman, University of California Press, Berkeley, California, 1999.

2. L. Lagrange, Mécanique Analytique, 1st Edition, Chez la Veuve Desaint, Paris, 1788; $2^{\text {nd }}$ Edition, Ve Courcier, Paris, 1811; $3^{\text {rd }}$ Edition, Mallet-Bachelier, Paris, 1853.

3. H. Goldstein, C.P. Poole, J.L. Safko, Classical Mechanics, $3^{\text {rd }}$ Edition, AddisonWesley, 2001.

4. A. Sinopoli, Il problema dell'equilibrio da Aristotele a Varignon, Franco Angeli, Milano, 2015.

5. T. Castellani, Equilibrio, Storia curiosa di un concetto fisico, Edizioni Dedalo, Bari, 2013.

6. Archimedes, On the equilibrium of planes, translated by J.L. Heiberg, Leipzig, 1910-15. 
7. S.C. Roero, La statica dall'arte alla scienza, Le Scienze, 1981: 150: 88-97.

8. Aristoteles, The Physics, translated by P.H. Wickstead and F.M. Cornford, Harvard University Press, Cambridge, Massachusetts, 1929.

9. R. Dugas, Histoire de la Mécanique, Éditions du Griffon, Neuchâtel, 1950.

10. G. Galilei, Della Scienza Meccanica, e della utilità che si sottraggono dagl'instrumenti di quella; opera del signor Galileo Galilei, con un frammento sopra la forza delle percossa, Ravenna, 1649.

11. Galileo Galilei, Discorsi e dimostrazioni matematiche intorno a due nuove scienze attinenti alla mecanica ed i movimenti locali, Leiden, 1638.

12. R.G. Newton, Galileo's Pendulum: From the Rhythm of Time to the Making of Matter, Harvard University Press, 2005.

13. P. Varignon, Nouvelle Mécanique ou Statique dont le projet fut donné en 1687, Ouvrage posthume de M. Varignon, C. Jombert, 1, Paris, 1725.

14. K. Culmann, Die graphisehe Statik, Verlag van Meyer \& Zeller, Zürich, 1864-66.

15. K.-E. Kurrer, The History of the Theory of Structures: From Arch Analysis to Computational Mechanics, Ernst \& Sohn, 2008.

16. L. Cremona, Graphical statics, two treatises on the graphical calculus, and reciprocal figures in graphical statics, Oxford Clarendon Press, 1890.

17. L. Euler, Methodus inveniendi lineas curvas maximi minimive proprietate gaudentes, sive solutio problematis isoperimetrici latissimo sensu accepti, Addentamentum 1: de curvis elasticis, Laussanae et Genevae, Apud MarcumMichaelem, Bousquet et Socios, 245-310, 1744.

18. W. Gautschi, Leonhard Euler: His Life, the Man, and His Works, Society for Industrial and Applied Mathematics (SIAM), 2008: 50: 3-33.

19. A.M. Lyapounov, Problème général de la stabilité du mouvement, Princeton University Press, Princeton NJ., 1949.

20. R. Thom, Structural Stability and Morphogenesis, An outline of a General Theory of Models, translated by D.H. Fowler, W.A. Benjamin, Inc., Advanced Book Program, Reading, Massachusetts, 1975.

21. Yu. A. Kuznetsov, Practical computation of normal forms on center manifolds at degenerate Bogdanov-Takens bifurcations, International Journal of Bifurcation and Chaos, 2005: 15:3535-3546.

22. P. Kowalczyk, M. di Bernardo, A.R. Champneys, S.J. Hogan, M. Homer, Yu. A. Kuznetsov, A. Nordmark, P.T. Piiroinen, Two-parameter nonsmooth bifurcations of limit cycles: classification and open problems, International Journal of Bifurcation and Chaos, 2006: 16(03): 601-629.

23. M. di Bernardo, C.J. Budd, A.R. Champneys, P. Kowalczyk, A.B. Nordmark, G.O. Tost, P.T. Piiroinen, Bifurcations in Nonsmooth Dynamical Systems, Society for Industrial and Applied Mathematics (SIAM), 2008: 50(4): 629-701.

24. S.P. Timoshenko, J.M. Gere, Theory of elastic stability, McGraw Hill, London, 1963.

25. A. Chajes, Principles of elastic stability, Prentice Hall, NJ, 1974.

26. J.M.T. Thompson, G.W. Hunt, A general theory of elastic stability, John Wiley, London, 1973. 
27. J.M.T. Thompson, The Elastic Instability of a Complete Spherical Shell, Aeronautical Quarterly, 1962: 13: 189-201.

28. Yu.A. Kuznetsov, Elements of Applied Bifurcation Theory, $2^{\text {nd }}$ Edition, Springer, New York, 1998.

29. Y. Chandraa, R. Wiebeb, I. Stanciulescu, L.N. Virginb, S.M. Spottswoodc, T.G. Easonc, Characterizing Dynamic Transitions Associated with Snap-Through of Clamped Shallow Arches, Journal of Sound and Vibration, 2013: 332: 5837-5855.

30. E. Hopf, Abzweigung einer periodischen Lösung von einer stationären Lösung eines Differentialsystems, Berichten der Mathematisch-Physischen Klasse der Sächsischen Akademie der Wissenschaften zu Leipzig, 1942: XCIV, 1-22. (An English translation, with comments, is included as Section 5 in [31]).

31. J.E. Marsden, M. McCracken, The Hopf Bifurcation and Its Applications, Springer-Verlag, New York, 1976.

32. D.H. Hodges, G.A. Pierce, Introduction to Structural Dynamics and Aeroelasticity, 2nd Edition, Cambridge University Press, 2011.

33. B.H.K. Lee, S.J. Price, Y.S. Wong, Nonlinear aeroelastic analysis of airfoils: bifurcation and chaos, Progress in Aerospace Sciences, 1999: 35: 205-334.

34. S. Rajasekar, M.A.F. Sanjuan, Nonlinear Resonances, Springer International Publishing, 2016.

35. E. Kartashova, Nonlinear Resonance Analysis, Cambridge University Press, 2010.

36. J. Guckenheimer, and P.J. Holmes, Nonlinear Oscillations, Dynamical Systems, and Bifurcations of Vector Fields, Springer-Verlag, New York, 1983.

37. S. Wiggins, Introduction to Applied Nonlinear Dynamical Systems and Chaos, Springer-Verlag, New York, 2003.

38. G. Floquet, Sur les équations différentielles linéaires à coefficients périodiques, Annales de l'École Normale Supérieure, 1883: 12, 47-88.

39. I.J.M. Besselink, Shimmy of Aircraft Main Landing Gears, PhD thesis, Delft University of Technology, 2000.

40. S.H. Strogatz, Nonlinear dynamics and chaos: with applications to physics, biology, chemistry and engineering, 2nd Edition, Westview Press, 2014.

41. J.M.T. Thompson, H.B. Stewart, Nonlinear dynamics and chaos, 2nd Edition, John Wiley \& Sons, England, 2002.

42. C. Grebogi, E. Ott, J.A. Yorke, Crises, sudden changes in chaotic attractors, and transient chaos, Physica D Nonlinear Phenomena, 1983: 7(1): 181-200.

43. W.T. Koiter, Over de stabiliteit van bet elastisch evenwicht, $\mathrm{PhD}$ thesis, Delft University, Delft, The Netherlands, 1945 (English transl.: W.T. Koiter, On the stability of elastic equilibrium, NASA technical translation F-10, 833, Clearinghouse, US Department of Commerce/National Bureau of Standards N67-25033, 1967).

44. S. Lenci, G. Rega, Load carrying capacity of systems within a global safety perspective. Part I. Robustness of stable equilibria under imperfections, International Journal of Non-Linear Mechanics, 2011: 46: 1232-1239.

45. A.M.A. van der Heijden, W.T. Koiter's Elastic Stability of Solid and Structures, Cambridge University Press, 2008. 
46. D. Bushnell, Buckling of Shells-Pitfall for Designers, AIAA JOURNAL, 1981: 19: $1183-1226$.

47. T.J.R. Hughes, J.A. Cottrell, Y. Bazilevs, Isogeometric analysis: CAD, finite elements, NURBS, exact geometry and mesh refinement, Computer Methods in Applied Mechanics and Engineering, 2005: 194: 4135-4195.

48. J.M.T. Thompson, Chaotic phenomena triggering the escape from a potential well, Proceedings of the Royal Society of London A, 1989: 421: 195-225.

49. M.S. Soliman, J.M.T. Thompson, Integrity measures quantifying the erosion of smooth and fractal basins of attraction, Journal of Sound and Vibration, 1989: 135: $453-475$.

50. J.M.T. Thompson, S.R. Bishop, L.M. Leung, Fractal basins and chaotic bifurcations prior to escape from a potential well, Physics Letters A, 1987: 121: 116-120.

51. S. Lenci, G. Rega, L. Ruzziconi, The dynamical integrity concept for interpreting/predicting experimental behaviour: from macro- to nano-mechanics, Philosophical Transaction of the Royal Society A, 2013: 371: 20120423.

52. S. Lenci, G. Rega, Load carrying capacity of systems within a global safety perspective. Part II. Attractor/basin integrity under dynamic excitations, International Journal of Non-Linear Mechanics 2011: 46: 1240-1251.

53. G. Rega, S. Lenci, Dynamical integrity and control of nonlinear mechanical oscillators, Journal of Vibration and Control, 2008: 14(1-2): 159-179.

54. F.M. Alsaleem, M.I. Younis, L. Ruzziconi, An experimental and theoretical investigation of dynamic pull-in in MEMS resonators actuated electrostatically, Journal of Microelectromechanical Systems, 2010: 19: 794-806.

55. L. Ruzziconi, A.H. Ramini, M.I. Younis, S. Lenci, Nonlinear Dynamics of an Electrically Actuated MEMS Device: Experimental and Theoretical Investigation, Sensors, 2014: 14: 17089-17111.

56. F.M. Alsaleem, M.I. Younis, H.M. Ouakad, On the nonlinear resonances and dynamic pull-in of electrostatically actuated resonators, Journal of Micromechanics and Microengineering, 2009: 19: 45013-46026.

57. F.M. Alsaleem, M.I. Younis, Theoretical and Experimental Investigation of Dynamic Instabilities in Electrostatic MEMS, Proceedings of the XIth International Congress and Exposition on Experimental and Applied Mechanics, Orlando, Florida, USA, June 2-5, 2008, Society for Experimental Mechanics (SEM), 2008.

58. G. Rega, S. Lenci, A global dynamics perspective for system safety from macroto nano-mechanics: Analysis, control and design engineering, Applied Mechanics Review, 2015: 67: 050802-1-19.

59. P. Belardinelli, S. Lenci, An efficient parallel implementation of Cell mapping methods for MDOF systems, Nonlinear Dynamics, 2016: 86: 2279-2290. 